\title{
Repercussões sociais da aquisição de uma deficiência física na vida de idosos
}

Social repercussions of the acquisition of a physical disability in elderly living

Repercusiones sociales de la adquisición de una discapacidad física en la vida de las personas mayores

Tatiane Dias Casimiro Valença Pollyanna Viana Lima Renato Novaes Chaves Elaine dos Santos Santana Luciana Araújo dos Reis

RESUMO: O objetivo foi analisar as repercussões sociais na vida de idosos que adquiriram deficiência física na velhice, identificando e discutindo as mudanças, desafios e adaptações que ocorreram após esta aquisição. Estudo exploratório, descritivo, com abordagem qualitativa realizado em um Centro Especializado em Reabilitação Física e Auditiva, com 22 idosos que apresentavam deficiência física adquirida, utilizando-se como instrumentos o questionário sociobiodemográfico e a entrevista. Para a análise dos dados, foi empregado o softwear NVivo e a análise de conteúdo temática. A aquisição da deficiência física na velhice gerou dependência, isolamento social, tristeza, afastamento do trabalho e preconceito, mas foi possível se adaptar e superar tal situação.

Palavras chave: Idoso; Pessoas com Deficiência Física; Adaptação.

ABSTRACT: The objective to know the social impact on the lives of older people who have acquired disabilities. A descriptive, exploratory study with a qualitative approach conducted in a Specialized Center for Physical Rehabilitation and Hearing with 22 elderly people who had acquired physical disability, using as instruments the sociobiodemográfico questionnaire and interview. For the analysis of data was used NVivo softwear and thematic content analysis. The acquisition of disability in old age generated dependency, social isolation, sadness, absence from work and prejudice, but it is possible to adapt and overcome such situation.

Keywords: Elderly; People with Physical Disabilities; Adaptación. 
RESUMEN: El objetivo fue conocer el impacto social en la vida de las personas mayores que han adquirido la discapacidad. Estudio exploratorio descriptivo, con enfoque cualitativo, realizado en un centro especializado para la rehabilitación física y la audición con 22 personas de edad avanzada que habían adquirido la discapacidad física utilizando como instrumentos sociobiodemográfico cuestionario y la entrevista. Para la análisis de los datos se utilizó NVivo softwear y análisis de contenido temático. La adquisición de la discapacidad en la vejez la dependencia generada, el aislamiento social, la tristeza, la ausencia del trabajo y los prejuicios, pero es posible adaptar y superar tal situación.

Palabras clave: Edad Avanzada; Las Personas con Discapacidades Físicas; Adaptação.

\section{Introdução}

Em vários países está ocorrendo um fenômeno demográfico caracterizado pelo rápido processo de envelhecimento populacional, principalmente, nos países em desenvolvimento (WHO, 2012). De acordo com estimativas do Instituto Brasileiro de Geografia e Estatística/ IBGE (2012), no Brasil a população de idosos deve passar de 14,9 milhões em 2013 para 58,4 milhões em 2060 (IBGE, 2012). A partir dessas projeções até 2025, o Brasil pode apresentar a sexta maior população de idosos em nível mundial (IBGE, 2012). Este cenário pode ser explicado pela associação de alguns fatores, como a diminuição das taxa de fecundidade, redução da mortalidade, ampliação da expectativa de vida ao envelhecer, melhoria dos serviços de saúde e o uso de novas tecnologias em geral (IBGE, 2012).

Ao mesmo tempo em que ocorre essa mudança demográfica também é observada, no Brasil, uma alteração no aspecto epidemiológico com o aumento da incidência das doenças crônico-degenerativas. Essas patologias afetam, principalmente, o sistema circulatório, apresentando-se na forma de Acidente Vascular Encefálico/AVE e Diabetes Mellitus. Elas estão diretamente relacionadas com a idade mais avançada, podendo ter como consequência a aquisição de uma deficiência física (Brasil, 2012a). De acordo com dados epidemiológicos mundiais mais de um bilhão de pessoas apresenta algum tipo de deficiência (WHO, 2012). No Brasil, no ano de 2010, 45,6 milhões de pessoas foram identificadas com pelo menos uma deficiência, o que corresponde a 23,9\% da população total do país, sendo que, deste número, 3 milhões de pessoas (7\%) apresentam a deficiência do tipo física (WHO, 2012).

O conceito de deficiência física pode ser descrito como qualquer alteração que impossibilita o desempenho esperado e adequado da função física de uma parte do corpo que foi afetada (Brasil, 2015). A terminologia utilizada para se referir à pessoa com deficiência ao longo dos tempos variou de acordo com os aspectos sociais, culturais e históricos. Ao longo do processo histórico, a deficiência era percebida a partir de uma concepção discriminatória e marginalizada (Baptista, \& Rigotti, 2014).

O envelhecer associado a uma deficiência física pode se apresentar como uma situação ainda mais complexa de ser enfrentada, e superada pelo idoso, pela família e sociedade. 
Isso porque, muitas vezes, estes fenômenos são vistos de forma negativa e preconceituosa (Dátilo, \& Marin, 2015). A pessoa idosa, ao adquirir uma deficiência física pode apresentar limitação na sua capacidade funcional, ou seja, esta nova situação enfrentada pelo indivíduo pode afetar a realização de suas atividades da vida diária, e das laborais, influenciando também na sua forma de pensar, agir. e se relacionar nos ambientes sociais aos quais ele pertence.

Este estudo se mostra relevante, pois, embora o Brasil seja um dos países que apresenta um crescimento elevado da população idosa com deficiência, é observado um número escasso de pesquisas voltadas para esse segmento populacional. Além disso, o profissional de saúde necessita conhecer de maneira mais abrangente todos os aspectos do viver que podem influenciar na condição de saúde e qualidade de vida das pessoas idosas com deficiência física, como também para o fortalecimento de ações de saúde pública.

Nesse contexto, o objetivo deste estudo é analisar as repercussões sociais na vida de idosos que adquiriram uma deficiência física na velhice, identificando e discutindo as mudanças, desafios e adaptações que ocorreram após esta aquisição.

\section{Método}

Este estudo foi extraído da tese de doutorado: "Envelhecer com deficiência física: memória e Representações Sociais de pessoas idosas”. Trata-se de um estudo exploratório, descritivo, com abordagem qualitativa, realizado em um Centro Especializado em Reabilitação Física e Auditiva, localizado no município de Vitória da Conquista, BA. Os participantes da pesquisa foram 22 idosos que atenderam aos seguintes critérios de inclusão: indivíduos com 60 anos ou mais de idade; que apresentavam algum tipo de deficiência física adquirida a partir dos 40 anos de idade; que estavam cadastradas no Centro de Reabilitação no período da coleta dos dados; apresentavam condições mentais preservadas para responder ao instrumento de pesquisa sendo avaliados através da aplicação do Mini-Exame do Estado Mental (Folstein, Folstein, \& McHugh, 1975), que apresentou, como ponto de corte, a pontuação $\geq$ de 19 pontos; e os que aceitaram participar voluntariamente da pesquisa.

Inicialmente foi solicitada uma autorização da Secretaria Municipal de Saúde para realização do estudo no Centro de Reabilitação. Com esta liberação foi realizada uma visita ao local para identificação dos participantes que atendiam aos critérios de inclusão, sendo agendados com o idoso o dia e o horário para a coleta das informações.

Em seguida, foi utilizado um questionário sociobiodemográfico, elaborado pelos pesquisadores, que buscava informações a respeito de: faixa etária, sexo, profissão, estado civil, renda, escolaridade, tipo e causa da deficiência física. Também foi empregada a entrevista semiestruturada (Bardin, 2011), composta por duas questões: 1) Como era a vida do(a) Senhor(a) antes de adquirir a deficiência física? 2) O que mudou na vida do(a) Senhor(a), após adquirir a deficiência física? 
A coleta dos dados foi realizada após as sessões de fisioterapia, de maneira individual em ambiente reservado no Centro de Reabilitação. O tempo de aplicação dos instrumentos de pesquisa variou de 40 até 60 minutos. Todas as entrevistas foram gravadas em áudio com autorização do entrevistado para que, posteriormente, fossem ouvidas e transcritas pelo pesquisador. A coleta de dados foi realizada durante o mês de agosto de 2015.

Para a análise dos dados, utilizou-se a técnica da análise de conteúdo temática, segundo Bardin (2011), na qual o tema é compreendido como a unidade de significação que se destaca do conteúdo do texto. Esta análise se baseia em três fases: a de pré-análise (leitura flutuante); a de exploração do material (com a codificação e categorização dos dados transformados em unidades de significação que descrevem as características do objeto analisado) e a de tratamento dos resultados coletados com a identificação das categorias temáticas (Bardin, 2011). As entrevistas foram codificadas por dois juízes de maneira individual e separada, concordando quanto às categorias que emergiram do corpus de resultados (Bardin, 2011).

A fim de auxiliar na realização do processo de análise do conteúdo, foi empregado o software QSR NVivo, versão 10, sendo este descrito como uma ferramenta informatizada destinada à codificação, tratamento, armazenamento e gerenciamento de arquivos de textos, vídeos, áudio entre outros, e apresentação gráfica de informações até então não estruturadas (Aranha, \& Gonçalves, 2007).

Este estudo cumpriu com os princípios éticos previstos na Resolução n. ${ }^{\circ} 466$, de 12 de dezembro de 2012, do Conselho Nacional de Saúde (Brasil, 2012b), tendo sido aprovado pelo Comitê de Ética em Pesquisa da Universidade Estadual do Sudoeste da Bahia, parecer n. ${ }^{\circ}$ 1.251.309. CAAE: 479159155.5.0000.0055. Ressalta-se que, para preservar o anonimato dos idosos participantes, estes foram identificados com siglas sendo a inicial maiúscula da palavra idoso seguida de um número (I1 até I22).

\section{Resultados}

A partir da análise do questionário sociobiodemográfico, foi possível traçar o perfil dos idosos entrevistados neste estudo. Constatou-se que 14 deles $(63,7 \%)$ se encontravam na faixa etária entre os 60 e 70 anos de idade; 19 (86,0\%) eram do gênero masculino e $13(59,1 \%)$ casados. Em relação à profissão que exerciam, 6 (27,3\%) trabalhavam na zona rural como agricultores; e 21(95,5\%) apresentavam como renda individual o valor entre 1 a 5 salários mínimos recebidos através do benefício social da aposentadoria junto ao Instituto Nacional do Seguro Social/INSS. Quanto ao nível de escolaridade, o maior número, 9 (40,9\%) possuíam o ensino fundamental II ( $6^{\circ}$ e o $9^{\circ}$ ano).

Em relação ao tipo de deficiência física, 17 (77,3\%) idosos apresentavam amputação de membro inferior, direito ou esquerdo, em diferentes níveis; e 5 (22,7\%) apresentavam hemiplegia como consequência de um AVE. No aspecto do fator causal da deficiência física, 10(45,5\%) dos idosos obtiveram tal aquisição por agravamento do quadro de trombose vascular periférica; e $5(22,7 \%)$, por AVE.

Valença, T. D. C., Lima, P. V., Chaves, R. N., Santana, E. dos S., \& Reis, L. A. dos. (2017, janeiro-março). Repercussões sociais da aquisição de uma deficiência física na vida de idosos. Revista Kairós Gerontologia, 20(1), pp. 41-55. ISSNe 2176-901X. São Paulo (SP), Brasil: FACHS/NEPE/PEPGG/PUC-SP 
Através da escuta, transcrição e análise do corpus construído a partir das falas dos idosos entrevistados emergiu a Categoria: Repercussões da aquisição da deficiência física na vida de idosos. Esta foi subdividida em duas subcategorias: 1. O viver antes de adquirir a deficiência física: atividade e trabalho; 2. O viver após adquirir a deficiência física: mudanças, desafios e adaptações. As falas referentes a cada subcategoria foram lançadas no software NVivo (Aranha \& Gonçalves, 2007) sendo possível a apresentação gráfica da frequência linguística que caracteriza cada subcategoria e apresentada na forma de nuvens de palavras.

- A vida antes de adquirir a deficiência física: atividade e trabalho

Os dez vocábulos que se mostraram mais evidentes nas falas dos idosos referentes a esta subcategoria foram: trabalhava, normal, trabalho, filhos, gostava, plantava, colhia, cuidava, família, festas. Estas foram apresentadas graficamente por meio da nuvem de palavras contidas na Figura 1.

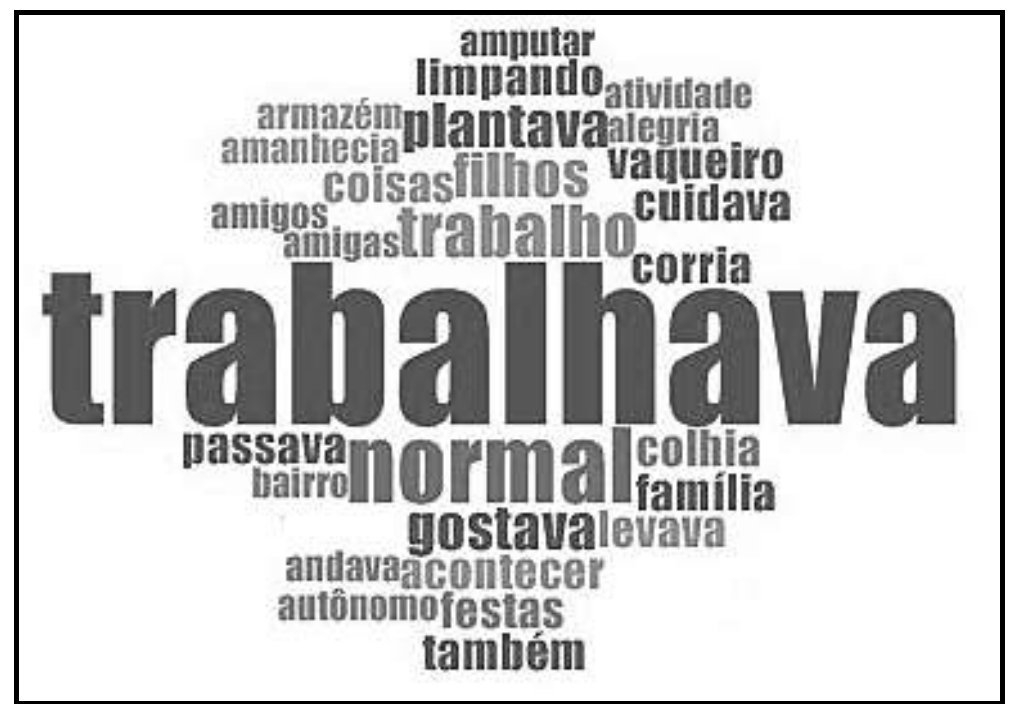

Figura 1: Nuvem de palavras "A vida antes de adquirir a deficiência física" Fonte: dados da pesquisa

As palavras que se apresentaram em maior frequência podem ser descritas como verbos que remetem a uma ação, evidenciando que estes idosos, antes de adquirirem a deficiência física, possuíam uma vida ativa e independente, como também foi observado nas falas a seguir:

"Eu sempre fui uma mulher ativa, que fazia tudo em casa. Gostava de cozinhar, limpava a casa, lavava roupa, fazia de tudo em casa." (I1)

“Eu pegava ônibus, ajudava nas coisas dentro de minha casa, fazia compra.”(I2)

As atividades da vida diária dessas pessoas idosas eram realizadas sem prejuízos e grandes limitações. As mulheres revelaram realizar os afazeres domésticos mesmo com a idade avançada, como também os homens realizavam ações corriqueiras como ir ao mercado, utilizar transporte público, dirigir e trabalhar. 
Em relação ao trabalho, foi constatado que grande parte dos entrevistados, principalmente do sexo masculino, ainda exerciam suas atividades laborais antes da aquisição da deficiência física e que o tipo de trabalho era pesado, no meio rural, ou em atividades mais desgastantes, como motorista de caminhão e vendedor.

"Eu trabalhava normal. Fazia tudo. Trabalhava o dia todo." (I2)

"Eu cuidava da roça. Plantava, molhava, limpava, colhia, era assim minha vida." (I5)

A respeito das atividades de lazer, os participantes relataram que estas eram mais frequentes antes da aquisição da deficiência física:

"Tinha meu lazer com os amigos, de ir para a venda, jogar conversa fora, jogar dominó, era assim, muito divertida." (I2)

"Antes de tudo acontecer, eu levava uma vida normal, saía para passear, para casa das amigas, ia nas festas. Era pouco que eu saía, mas ainda saía.”(I6)

- $\quad$ A vida após adquirir a deficiência física: mudanças, desafios e adaptações

Na Figura 2, temos uma imagem gráfica da nuvem de palavras com os dez vocábulos que apareceram com maior frequência nas falas dos idosos: trabalhar, difícil, prótese, desafio, preconceito, trabalho, dificuldade, acostumar, adaptar, movimento.

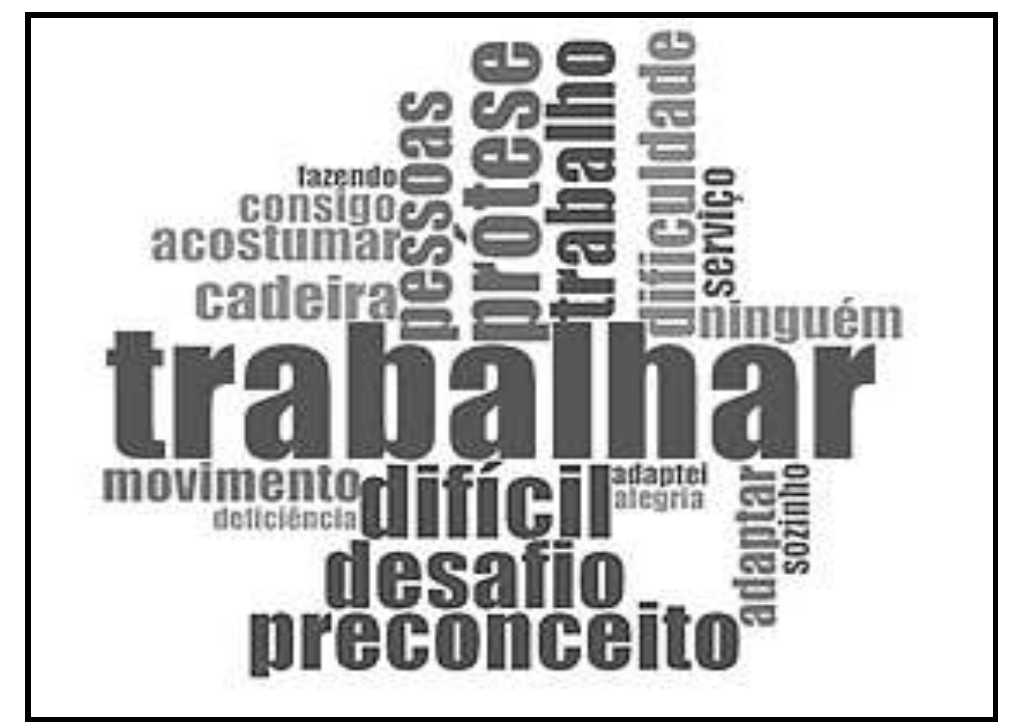

Figura 2: Nuvem de palavras "A vida após adquirir a deficiência física" Fonte: dados da pesquisa

A percepção de alguns idosos a respeito da chegada da deficiência física é que trouxe limitação ou incapacidade funcional para a realização de várias de suas atividades de vida diária, como a higiene pessoal, vestir-se, alimentar-se e locomover-se. A independência funcional, antes presente, deu lugar à dependência parcial ou total a outras pessoas, para cumprir as atividades cotidianas. 


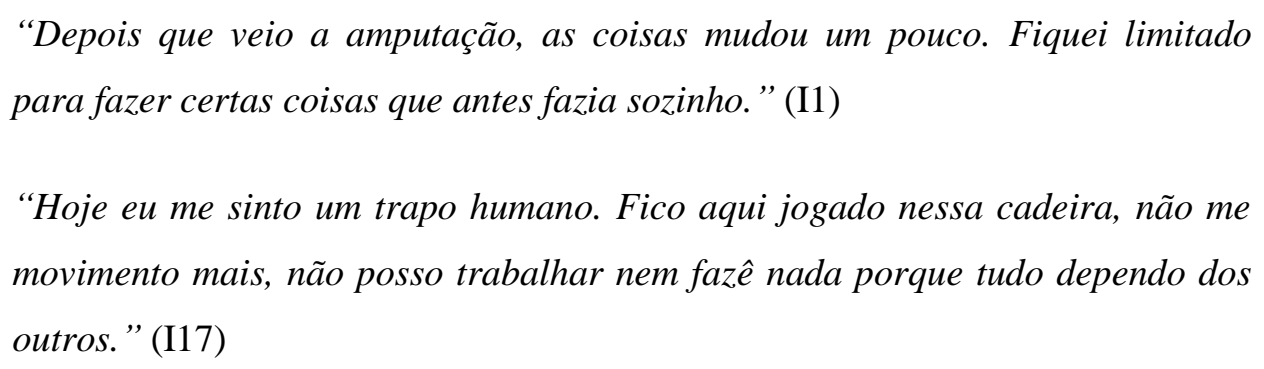

Com a aquisição da deficiência física, muitos idosos tiveram que abandonar sua atividade laboral, deixando de ser o provedor principal do lar, e tendo que aderir ao sistema de aposentadoria para suprir suas necessidades e da família:

“Às vezes, eu sinto falta do trabalho, porque fico dentro de casa muito tempo e era acostumado trabalhar. Se não tivesse amputado a perna, eu tava continuando a trabalhar." (19)

"Então, depois disso (AVE), eu tive que aposentar. Aposentei por invalidez $e$ hoje vivo com um salário." (I11)

Os participantes também declaram que, após a aquisição da deficiência física, eles vivenciaram situações preconceituosas por parte da sociedade e por pessoas da própria família, sendo consideradas pessoas dependentes e incapazes.

"O preconceito também é outra dificuldade. As pessoas te olham com pena ou acham que você acabou e que não presta pra nada." (I10)

No entanto, alguns idosos entrevistados se mostraram resilientes, mesmo diante das dificuldades e desafios enfrentados com tal aquisição. Esses idosos assumiram a identidade de deficiente físico, revelando uma maneira de pensar e agir de adaptação e superação:

"Depois do AVE, eu continuo trabalhando, dá pra trabalhar ainda, fico ajudando nas vendas, orientando as coisas, os vendedores. Tenho que ir lá porque é meu o negócio." (I21)

"Tem que enfrentar tudo que aparece na vida da gente. Não pode ficar com medo e ficar reclamando, não. Com perna ou sem perna, tá tudo bem. Ainda tenho saúde pra viver muitos anos ainda." (I6)

Foi observado que a volta ao trabalho é uma das expectativas de alguns idosos. A reabilitação física e o uso da prótese são vislumbrados como uma forma de adquirirem maior independência funcional e garantia de manutenção de seu papel social.

"O desafio é voltar a ter meus movimentos de volta e poder voltar a andar e trabalhar, isso é meu desafio maior. Mas, vou buscar voltar a trabalhar. Homem que é homem tem que trabalhar e sustentar a casa, já dizia meu avô." 


\section{Discussão}

Os idosos participantes deste estudo foram unânimes em descrever a aquisição da deficiência física na velhice como um acontecimento difícil de ser aceito e superado por eles. Esta aquisição provocou mudanças profundas que vão além da questão fisiológica, afetando também os aspectos social, econômico, psicológico, do seu viver. A partir dessa observação, podemos dizer que a definição de deficiência ainda é desafiadora, uma vez que engloba vários elementos que se relacionam de maneira complexa e dinâmica, e sofrem influência do processo histórico e social. De acordo com Nogueira, et al., (2016), é preciso analisar de maneira individual as pessoas com deficiência física, pois cada um possui uma história de vida única e coletivamente compartilhada.

Assim, além da capacidade individual de aceitar e superar problemas, a sociedade em que esse indivíduo está inserido pode influenciar com maior ou menor intensidade nas possibilidades de enfrentamento das limitações e dificuldades enfrentadas (Nogueira, et al., 2016), com a aquisição de uma deficiência física na velhice. Para Quaresma e Ribeirinho (2016), a eficácia dos sistemas de proteção social, como a elaboração e aplicação efetiva de políticas públicas constitui um elemento importante para o futuro do envelhecimento humano, principalmente se este estiver atrelado a uma deficiência física.

Foi observado na análise das informações obtidas que, ao adquirir a deficiência física, o idoso se deparou com a limitação ou incapacidade funcional para a realização das atividades da vida diária, e as laborais, e isso foi o motivo para o surgimento de sentimentos de vergonha, tristeza, insegurança, ansiedade, medo e incertezas, convergindo em quadros de depressão e exclusão do convívio social (Queiroz, et al., 2016). Em estudo realizado com pessoas com deficiência física foi constatado que $23,3 \%$ da amostra já tinha se afastado do convívio de grupos sociais de que participava, devido principalmente à dificuldade de locomoção (Holanda, et al., 2015). Com o isolamento social, o ócio e a solidão, os idosos podem desenvolver um quadro cada vez maior de tristeza e depressão, aumentando ainda mais o seu sofrimento (Queiroz, et al., 2016; Rodrigues, Aoki, \& Oliver, 2015; Aoki, \& Oliver, 2013).

Também foi observada em outra pesquisa, desenvolvida por Seren e De Tilio (2014), a presença de comportamentos agressivos, isolamento social, perda da auto-estima e impacto na identidade das pessoas com deficiência física. No estudo sobre redes de apoio à pessoa com deficiência física, Cruz, et al., (2015) concluíram que é importante que essas pessoas mantenham suas relações sociais e estabeleçam novas relações interpessoais, pois em algum momento podem necessitar do apoio e suporte que as ajudem a superarem suas dificuldades emocionais ou instrumentais da vida diária.

Conforme exposto por Ribeiro, Freitas, \& Souza (2016), a presença de morbidades e comorbidades que aparecem com o avançar da idade podem provocar prejuízos emocionais para a pessoa idosa. 
É consenso entre diversos autores que o convívio social contribui para o bem-estar; que, através das relações sociais, é possível promover melhores condições de saúde nos âmbitos físico e psicológico na vida das pessoas idosas (Wrzus, Hänel, Wagner, \& Neyer, 2013; Ribeiro, Freitas, \& Souza, 2016), e que a ausência do convívio social pode ocasionar diversos efeitos negativos para o idoso (Carneiro, 2014, Ribeiro, Freitas, \& Souza, 2016).

Pesquisa realizada com idosas dependentes no município de Cascavel, PR, revelou que muitas atividades que antes elas conseguiam executar sozinhas, como as tarefas relacionadas à sua higiene pessoal e alimentação, passaram a ser realizadas, ou elas próprias auxiliadas, por outras pessoas. Também foi observada uma nostalgia nas falas destas idosas, ao se relembrarem de sua autonomia perdida com a chegada da deficiência física (Queiroz, et al., 2016).

A situação de dependência funcional pode provocar o aparecimento de alterações psicológicas como: tristeza, apatia, culpa, tornando estas pessoas irritadas, intolerantes e ansiosas.

Em estudo realizado com idosos que realizaram amputação por causa do Diabetes Mellitus, concluiu-se que o indivíduo amputado relata ter vivenciando uma tragédia, uma vez que a experiência da mutilação é devastadora, sendo mencionados, com frequência por estas pessoas, sintomas depressivos como tristeza, pesar, episódios de choro, isolamento social, perda de apetite, dificuldade para dormir, inferioridade em relação às outras pessoas e baixa da auto-estima (Bello, Souza, Comassetto, \& Oliveira, 2014).

Em relação à qualidade de vida o estudo realizado com indivíduos que tiveram AVE, e que dependiam dos cuidadores para as atividades de vida diária, revelou que estes indivíduos exibiam pior qualidade de vida em relação aos que não apresentaram sequelas, como a hemiplegia. Foi identificado que 49,7\% destes participantes apresentaram sintomas depressivos evidentes devidos, principalmente, à limitação funcional (Rangel, Belasco, \& Diccini, 2013). Ou seja, a perda da independência e autonomia compromete diretamente a qualidade de vida do indivíduo idoso.

Além do aspecto funcional e emocional, os idosos com deficiência física também vivenciam problemas de acessibilidade. Mesmo os idosos mais independentes funcionalmente têm dificuldades de acesso aos espaços urbanos e transporte público adaptado, uma vez que estes foram planejados considerando necessidades destes indivíduos (Kikuchi, et al., 2014). Com isso, os idosos ficam isolados em seus lares, deixando de manter suas relações sociais, aumentandolhes a solidão e a depressão. Desse modo, seus direitos como cidadão não são respeitados, nem atendidos pela sociedade.

Com a aquisição da deficiência física, os idosos apresentaram dificuldades de locomoção e de realizarem determinados movimentos, provocando o afastamento forçado da sua atividade profissional. Esta situação foi descrita como algo que provocou dúvidas, preocupação e medo em relação a seu sustento e da família.

Desse modo, a renda familiar sofreu uma redução, afetando o padrão e a qualidade de vida do idoso e de sua família. Na maioria das vezes, estes indivíduos passam a receber uma aposentadoria. No entanto, muitos relataram que o valor recebido é escasso em relação ao valor gasto com alimentação, medicamentos e outras despesas básicas (Holanda, et al., 2015). 
Para alguns idosos, o papel de mantenedor do lar era motivo de orgulho e essa nova situação é representada como uma perda de seu papel na família e na sociedade (Holanda, et al., 2015).

Ficou evidenciado, nas duas nuvens de palavras, o termo "trabalhar", revelando que, antes ou depois da aquisição da deficiência física, esta atividade está presente na sua vida, seja como uma lembrança, seja como uma perspectiva futura. $\mathrm{O}$ afastamento das atividades laborais contribuiu para que estes idosos se sentissem incapazes, sozinhos e desvalorizados. Ou seja, o fato de viverem em uma sociedade capitalista, que é voltada para a produção de riquezas, e a condição de não estarem mais aptos a realizar as atividades laborais tal como antes da aquisição da deficiência física, provocaram confusões em suas realidades e imaginários (Naressi, GirardonPerlini, Sand, Beuter, \& Rosa, 2013). Assim, o trabalho se mostra como um importante aspecto da vida do idoso que pode contribuir para as relações e trocas sociais, evitando a exclusão e a solidão (Holanda, et al., 2015; Zołnierczyk-Zreda, \& Majewski, 2012).

Outra situação descrita pelos idosos foi o preconceito. O envelhecimento é percebido pela sociedade por um ponto de vista depreciativo. Associando-se o envelhecer a uma deficiência física, estamos diante de uma visão duplamente estigmatizante por parte da sociedade (Giacomin, \& Firmo, 2015). Nos dias atuais, existe uma busca pela beleza e juventude eterna, com um culto exagerado ao corpo e à perfeição física. As pessoas que apresentam algum tipo de deficiência física são postos à marginalidade, sofrendo duplamente, seja por meio de obstáculos públicos que impedem sua locomoção, seja por serem considerados incapacitados (Garbe, 2013).

Assim, as concepções que prevalecem sobre o envelhecimento e a deficiência física são de declínio físico, inutilidade, diferença e perda do papel social (Santos, Tura, \& Arruda, 2013), oO que pode acarretar sentimentos de piedade pela sociedade. Essa maneira de perceber a deficiência física pode refletir uma situação de estigma, uma vez que permite a formação de julgamentos tácitos e antecipados sobre o status moral e a identidade da pessoa com deficiência (Goffman, 1988; Barsaglini, \& Biato, 2015). Situações estigmatizantes são descritas pelos participantes deste e de outros estudos como experiências marcantes que podem ter, como consequência, o isolamento social e a depressão (Holanda, et al., 2015; Santos, Tura, \& Arruda, 2013; Martins, \& Barsaglini, 2011).

No entanto, mesmo a aquisição da deficiência física sendo relatada como uma situação difícil de ser enfrentada, a maioria dos idosos deste estudo se mostrou resiliente, demonstrando auto-aceitação de sua imagem corporal e da limitação funcional imposta pela deficiência física (Sabino, Torquato, \& Pardini, 2016). Além disso, alguns participantes revelaram que a limitação funcional não implica em empecilho para pensar, tomar decisões sobre sua vida e fazer planos futuros. De acordo com o estudo, muitas vezes a limitação física é confundida com a impossibilidade para tomada de decisão (Mirandol, \& Bós, 2015). Todavia, é fundamental desvincular as perdas físicas das cognitivas, pois os com deficiência física apresentam limitações funcionais e não cognitivas; sendo assim, capazes de tomar decisões sobre sua vida. 
Foi possível observar que os participantes deste estudo, mesmo vivenciando situações de perdas funcionais significativas, apresentaram sentimentos positivos, nos quais é enfatizada a adaptação e superação através da reabilitação física e do apoio da família (Brito, Oliveira, \& Eulalio, 2015). O acesso aos serviços de fisioterapia, segundo a literatura, promove ganhos funcionais, auxilia na reinserção social da pessoa com deficiência, e na readaptação às atividades da vida diária (Mendes, et al., 2016).

Nessa perspectiva, o idoso que adquiriu uma deficiência física na velhice pode se tornar resiliente, aceitando sua condição, adaptando-se e superando as limitações físicas, sociais e psicológicas assim impostas, procurando transpor os obstáculos e esboçando novas metas pessoais (Batista, \& Luz, 2012). A resiliência pode ser descrita como a capacidade que o indivíduo apresenta de enfrentar, vencer e ser fortalecido ou transformado por experiências de adversidade, ou seja, pela sua capacidade de autorregulação e auto-estima (Naressi, et al., 2013).

Entretanto, para vencerem os desafios e superarem os obstáculos advindos com a deficiência física, eles precisam contar com ajuda de uma rede de apoio, como a família, a sociedade e o Estado, buscando atender as necessidades e os direitos dessa parcela da população que cresce de maneira rápida e intensa no Brasil. Foi observado, na literatura (Rodrigues, \& Ferreira, 2012; Barbosa, Balieiro, \& Pettengill, 2012; Cruz, et al., 2015), que pessoas com deficiência física, necessitam de auxílio de maneira geral ou em algumas atividades específicas como higiene pessoal e locomoção, incumbindo a família de suprir essas demandas de cuidado. No entanto, nem sempre a família está preparada para assumir tal incumbência, sendo necessário adaptar-se à realidade imposta (Barbosa, et al., 2012).

Finalmente, destaca-se que os resultados deste estudo não revelam resultados generalizados referentes a todos os idosos que adquiriram uma deficiência física. Portanto, é necessária a realização de outras pesquisas que abordem o tema em diferentes contextos regionais, sociais, econômicos e culturais para se alcançar resultados mais abrangentes.

\section{Considerações finais}

O envelhecimento humano é hoje uma realidade mundial. O Brasil está envelhecendo a passos largos e também vem apresentando um número elevado de pessoas idosas com deficiência física. Esta aquisição, principalmente na fase da velhice pode trazer transtornos, desafios e limitações funcionais que vão influenciar na forma de viver destes indivíduos.

Neste estudo, foi possível conhecer as experiências sociais vivenciadas pelo idoso antes e após a aquisição da deficiência física. $\mathrm{O}$ viver anterior a esta aquisição se revela condizente com o processo natural de envelhecimento humano ativo, no qual se observa algumas limitações próprias dessa fase da vida, mas uma autonomia funcional que ainda permite a realização das atividades da vida diária e laborais.

No entanto, a aquisição de uma deficiência física vai afetar esse processo, gerando dependência funcional, que pode variar de acordo com o tipo e grau de deficiência. Atividades do cotidiano são realizadas de forma limitada ou são impedidas de serem executadas. 
As relações sociais com a família, lazer, e trabalho, são reduzidas ou abolidas, podendo, dessa forma, contribuir para o isolamento social e a depressão.

Mas alguns destes idosos demonstram adaptação, superação e resiliência em relação a esta aquisição. A busca pela reabilitação, a vontade de voltar a ser ativo, trabalhar, se relacionar com outras pessoas e ser feliz foi identificadas nos relatos destes idosos, demonstrando esperança em melhorar sua condição funcional e voltar a ser independente.

Nesse contexto, mostra-se fundamental para o profissional da área de saúde buscar compreender para além do aspecto físico da aquisição da deficiência física. É necessário verificar como este idoso percebe e busca ultrapassar os obstáculos sociais, econômicos e psicológicos dessa nova forma de viver. A partir desse conhecimento, estes profissionais poderão alcançar uma visão mais abrangente de todos os contextos que envolvem esta aquisição, para então, desenvolver ações que busquem atender as necessidades dessa população, contribuindo para sua maior independência funcional e qualidade de vida.

\section{Referências}

Aoki, M., \& Oliver, F. C. (2013). Pessoas com deficiência moradoras de bairro periférico da cidade de São Paulo: estudo de suas necessidades. Cad Ter Ocup UFSCar [Internet], 21(2), 391398. Recuperado em 22 setembro, 2016, de:

http://www.cadernosdeterapiaocupacional.ufscar.br/index.php/cadernos/article/view/825.

Aranha, A., \& Gonçalves, F. (2007). Métodos de análise de conteúdo: NUD*IST (non-numerical unstructed data indexing, searching, and theorizing), Nvivo7. Vila Real: UTAD. (27 p.).

Batista, N. N. L. A. L., \& Luz, M. H. B. A. (2012). Vivências de pessoas com diabetes e amputação de membros. Rev Bras Enferm [Internet], 65(2), 244-250. Recuperado em 12 agosto, 2016, de: http://www.scielo.br/scielo.php?script=sci_arttext\&pid=S0034-71672012000200007.

Baptista, E. A., \& Rigotti, J. I. R. (2014). Minas Gerais e sua população de deficientes: um estudo a partir dos Censos Demográficos de 2000 e 2010. Caderno de Geografia [Internet], 24(41), 98118. Recuperado em 13 agosto, 2016, de:

http://periodicos.pucminas.br/index.php/geografia/article/view/6327.

Bardin, L. (2011). Análise de conteúdo. (2ª ed.). São Paulo, SP: Edições 70. (229 p.).

Barbosa, M. A. M., Balieiro, M. M. F. G., \& Pettengill, M. A. M. (2012). Cuidado centrado na família no contexto da criança com deficiência e sua família: uma análise reflexiva. Texto Contexto-Enferm., 21(1), 194-199. Recuperado em 13 agosto, 2016, de: http://periodicos.pucminas.br/index.php/geografia/article/view/6327.

Baptista, E. A., \& Rigotti, J. I. R. (2014). Minas Gerais e sua população de deficientes: um estudo a partir dos Censos Demográficos de 2000 e 2010. Caderno de Geografia, 24(41), 98-118. Recuperado em 10 agosto, 2016, de: http://www.redalyc.org/pdf/3332/333229407008.pdf.

Barsaglini, R. A., \& Biato, E. C. L. (2015). Compaixão, piedade e deficiência física: o valor da diferença nas relações heterogêneas. História, Ciências, Saúde-Manguinhos, 22(3), 781-796. Recuperado em 13 agosto, 2016, de: http://www.revistahcsm.coc.fiocruz.br/compaixao-piedadee-deficiencia-fisica/.

Bello, E. F., Souza, E. M., Comassetto, I., \& Oliveira, J. M. (2014). Experience of the institutionalized elderly with amputated lower limbs due to complications of diabetes mellitus. $J$ Nurs UFPE [Internet], 8(1), 44-51. Recuperado em 10 agosto, 2016, de: http://www.revista.ufpe.br/revistaenfermagem/index.php/revista/article/view/4158/pdf_4400. 
Brasil. (2012a). Cartilha do Censo 2010: Pessoas com Deficiência. Luiza Maria Borges Oliveira. Secretaria de Direitos Humanos da Presidência da República /SDH/PR. Secretaria Nacional de Promoção dos Direitos da Pessoa com Deficiência /SNPD. Coordenação-Geral do Sistema de Informações sobre a Pessoa com Deficiência; Brasília, DF: SDH PR/SNPD, 2012. (32 p.). Recuperado em 10 agosto, 2016, de: http://www.pessoacomdeficiencia.gov.br/app/.

Brasil. (2012b). Conselho Nacional de Saúde. Resolução 466, de 12 de dezembro de 2012. [Internet]. Recuperado em 10 agosto, 2016, de: http://conselho.saude.gov.br/resolucoes/2012/Reso466.pdf.

Brasil. (2015). Lei n. ${ }^{\circ} 13.146$, de 6 de julho de 2015. Lei Brasileira de Inclusão da Pessoa com Deficiência. Estatuto da Pessoa com Deficiência. Recuperado em 22 maio, 2016, de: http://www.planalto.gov.br/ccivil_03/_ato2015-2018/2015/Lei/L13146.htm.

Bello, E. F., Souza, E. M., Comassetto, I., \& Oliveira, J. M. (2014). Experience of the institutionalized elderly with amputated lower limbs due to complications of diabetes mellitus. Journal of Nursing UFPE on line, 8(1), 44-51. Recuperado em 01 julho, 2015, de: doi: 10.5205/reuol.4843-39594-1-SM.0801201407.

Brito, R. D. Q., Oliveira, A. R., \& Eulalio, M. C. (2015). Deficiência física e envelhecimento: estudo das representações sociais de idosos sob reabilitação fisioterápica. Av Psicol Latinoam [Internet], 33(1), 121-33. Recuperado em 08 de setembro, 2016, de: http://www.scielo.org.co/pdf/apl/v33n1/v33n1a09.pdf.

Carneiro, R. S. (2014). Um estudo das habilidades sociais em idosos. Psicologia Argumento, 32(76), 23-31. Recuperado em 08 setembro 2016, de:

http://www2.pucpr.br/reol/pb/index.php/pa?dd1=14559\&dd99=view\&dd98=pb.

Cruz, D. M., Nascimento, L. R. S., Silva, D. M. G. V., \& Dornelles, S. S. (2015). Redes de apoio à pessoa com deficiência física. Ciencia y enfermería, 21(1), 23-33. Recuperado em 22 junho, 2016, de: https://dx.doi.org/10.4067/S0717-95532015000100003.

Dátilo, G. M. P. A., \& Marin, M. J. S. (2015). O envelhecimento na percepção de idosos que frequentam uma Universidade Aberta da Terceira Idade. Estud interdiscipl envelhec [Internet], 20(2), 597-609. Recuperado em 22 junho, 2016, de: http://www.seer.ufrgs.br/ RevEnvelhecer/article/view/48932.

Folstein, M. F., Folstein, S. E., \& McHugh, P. R. (1975). Mini-Mental State: a practical method for grading the cognitive state of patients for clinician. J Psychiatr Res [Internet], 12, 189-198. Recuperado em 03 junho, 2016, de: http://www.journalofpsychiatricresearch.com/article/00223956(75)90026-6/abstract.

Garbe, D. S. (2012). Acessibilidade às pessoas com deficiência física e a Convenção Internacional de Nova Iorque. Revista da UNIFEBE [Internet], 10, 95-104. Recuperado em 17 agosto, 2016, de: http://periodicos.unifebe.edu.br/index.php/revistaeletronicadaunifebe/article/ view/3/2.

Giacomin, K. C., \& Firmo, J. O. A. (2015).Old age, disability and care in public health. Ciênc Saúde Coletiva [Internet], 20(12), 3631-3640. Recuperado em 29 agosto, 2016, de: http://www.scielo.br/pdf/csc/v20n12/en_1413-8123-csc-20-12-3631.pdf.

Goffman, E. (1988). Estigma: notas sobre a manipulação da identidade deteriorada. (4a ed.). Rio de Janeiro, RJ: Livros Técnicos e Científicos (124 p.).

Holanda, C. M. A., Andrade, F. L. J. P., Bezerra, M. A., Nascimento, J. P. S., Neves, R. F., Alves, S. B. \& Ribeiro, K. S. Q. S. (2015). Support networks and people with physical disabilities: social inclusion and access to health services. Ciênc Saúde Colet [Internet], 20(1), 175-184. Recuperado em 29 maio, 2016, de:

http://www.scielo.br/scielo.php?script=sci_arttext\&pid=S1413-81232015000100175.

Instituto Brasileiro de Geografia e Estatística/IBGE. (2012). Comunicação Social. Índice de envelhecimento no Brasil cresce de 31,7, em 2001, para 51,8 em 2011. In: SIS 2012: Acesso de jovens pretos e pardos à universidade triplicou em dez anos. Rio de Janeiro, RJ: IBGE, 28 nov. 2012. Recuperado em 29 maio, 2016, de: http://saladeimprensa.ibge.gov.br/noticias?id=1\& idnoticia=2268\&t=sis-2012-acesso-jovens-pretos-pardos-universidade-triplicou-dez-anos\&view=noticia.

Kikuchi, E., Maturana, C. S., Bispo, N. N. C., Costa Filho, R. M., Lopes, R., Concone, M. H. V. B., \& Probst, V. S. (2014). O significado das limitações dos idosos para estudantes de Fisioterapia. Revista Kairós Gerontologia [Internet], 17(4), 95-118. Recuperado em 02 outubro, 2016, de: http://revistas.pucsp.br/index.php/kairos/article/view/24058/17307. 
Martins, J. A., \& Barsaglini, R. A. (2011). Aspectos da identidade na experiência da deficiência física: um olhar socioantropológico. Interface [Internet],15(36), 109-122. Recuperado em 13 outubro, 2016, de: http://www.scielosp.org/pdf/icse/v15n36/aop4110.pdf.

Mendes, L. M., Gadelha, I. D. S., Brito, G. E. G., Moraes, R. M., \& Ribeiro, K. S. Q. S. R. (2016). Individuals' access after cerebrovascular accident to the physical therapy services. J Nurs UFPE [Internet], 10(2), 387-394. Recuperado em 01 novembro, 2016, de: http://www.revista.ufpe.br/revistaenfermagem/index.php/revista/article/view/8559/pdf_9505.

Mirandola, A. R., \& Bós, A. J. G. (2015). Relação entre capacidade funcional e capacidade de tomada de decisão em longevos. PAJAR, 3(2), 53-59. Recuperado em 01 novembro, 2016, de: http://revistaseletronicas.pucrs.br/ojs/index.php/pajar/article/view/22532.

Naressi, D. A., Girardon-Perlini, N. M. O., Sand, I. C. P., Beuter, M., \& Rosa, B. V. C. (2013). Beliefs and resilience in patients who are survivors of leukemia. J Nurs UFPE [Internet], 7(1), 67-75. Recuperado em 13 setembro, 2016, de: http://www.revista.ufpe.br/revistaenfermagem/ index.php/revista/article/viewFile/3253/pdf_1813.

Nogueira, G. C., Schoeller, S. D., Ramos, F. R. S., Padilha, M. I., Brehmer, L. C. F., \& Marques, A. M. F. B. (2016). Perfil das pessoas com deficiência física e Políticas Públicas: a distância entre intenções e gestos. Ciência \& Saúde Coletiva, 21(10), 3131-3142. Recuperado em 23 novembro, 2016, de: https://dx.doi.org/10.1590/1413-812320152110.17622016.

Quaresma, M. L., \& Ribeirinho, C. (2016). Envelhecimento - Desafios do Séc. XXI. Revista Kairós Gerontologia, 19(3), 29-49. Recuperado em 01 novembro, 2016, de: http://revistas.pucsp.br/index.php/kairos/article/view/30900/21382.

Queiroz, A. A. F. L. N., Morais, E. R., Silva, R. A. F., Guimaraes, M. S. O., Oliveira, L. B., \& Magalhães, R. L. B. (2016). Vivências de vítimas de amputação por acidentes. J Nurs UFPE [Internet], 10(Supl. 2), 708-713. Recuperado em 28 novembro, 2016, de: http://www.revista.ufpe.br/revistaenfermagem/index.php/revista/article/view/8707/pdf_9669.

Rangel, E. S. S., Belasco, A. G. S., \& Diccini, S. (2013). Quality of life of patients with stroke rehabilitation Acta Paul Enferm [Internet], 26(2), 205-212. Recuperado em 16 setembro, 2016, de: http://www.scielo.br/pdf/ape/v26n2/en_v26n2a16.pdf.

Ribeiro, P. C. C., Freitas, V. J., \& Souza, J. S. (2016). A busca pelo atendimento psicológico na meia-idade e na velhice. Revista Kairós Gerontologia, 19(2), 65-83. Recuperado em 28 novembro, 2016 de: http://revistas.pucsp.br/index.php/kairos/article/view/27409/20787.

Rodrigues, J. S. M., \& Ferreira, N. M. L. A. (2012). Estrutura e funcionalidade da rede de apoio social do adulto com câncer. Acta Paul Enferm., 25(5), 781-787. Recuperado em 01 novembro, 2016, de: http://www.chs.saude.sp.gov.br/resources/humanizacao/biblioteca/artigoscientificos/a004_estrutura_e _funcionalidade_da_rede_de_apoio_social_do_adulto_com_cancer_-_acta_2012.pdf.

Rodrigues, S. M., Aoki, M., \& Oliver, F. C. (2015). Diagnóstico situacional de pessoas com deficiência acompanhadas em terapia ocupacional em uma unidade básica de saúde. Cad Ter Ocup UFSCar [Internet], 23(4), 781-794. Recuperado em 01 novembro, 2016 de: http://www.cadernosdeterapiaocupacional.ufscar.br/index.php/cadernos/article/view/1352/666.

Sabino, S. D. M., Torquato, R. M., \& Pardini, A. C. G. (2013). Ansiedade, depressão e desesperança em pacientes amputados de membros inferiores. Acta Fisiátr [Internet], 20(4), 224-228. Recuperado em 23 setembro, 2016, de: http://www.actafisiatrica.org.br/detalhe_artigo.asp?id=525.

Santos, V. B., Tura, L. F. R., \& Arruda, A. M. S. (2013). As representações sociais de "pessoa velha" construídas por idosos. Saúde Soc [Internet], 22(1), 138-147. Recuperado em 01 novembro, 2016, de: http://www.scielo.br/pdf/sausoc/v22n1/13.pdf.

Seren, R., \& De Tilio, R. (2014). As vivências do luto e seus estágios em pessoas amputadas. Revista da SPAGESP [Internet], 15(1), 64-78. Recuperado em 01 novembro, 2016, de: http://pepsic.bvsalud.org/pdf/rspagesp/v15n1/v15n1a06.pdf.

Zołnierczyk-Zreda, D., \& Majewski, T. (2012). Adaptation of the working environment to the capacities of workers with physical, intellectual and mental disabilities. Med Pr., 63(4), 493-504. Recuperado em 26 de outubro, 2016, de: https://www.ncbi.nlm.nih.gov/pubmed/22994079.

World Health Organization/WHO. World Report on Disability. (2011). Recuperado em 01 novembro, 2016 de: http://www.who.int/disabilities/world_report/2011/report.pdf. 
WHO. (2012). World Health Organization. Knowledge translation on ageing and health: $a$ framework for policy development. Geneve, Suisse: WHO [Internet]. Recuperado em 01 novembro, 2016 de: http://www.who.int/ageing/publications/knowledge_translation_en.pdf.

Wrzus, C., Hänel, M., Wagner, J., \& Neyer, F. J. (2013). Social network changes and life events across the life span: A meta-analysis. Psychological Bulletin, 139(1), 53-80. Recuperado em 01 março, 2015, de: https://www.ncbi.nlm.nih.gov/pubmed/22642230.

Recebido em 12/12/2016

Aceito em 30/01/2017

Tatiane Dias Casimiro Valença - Fisioterapeuta, Doutoranda do Programa de Pós-Graduação em Memória: Linguagem e Sociedade da Universidade Estadual do Sudoeste da Bahia/ PPGM/UESB, Docente Assistente do Curso de Fisioterapia da Universidade Estadual do Sudoeste da Bahia /UESB.

E-mail: tativalenca26@gmail.com

Pollyanna Viana Lima - Enfermeira, Doutoranda do Programa de Pós-Graduação em Memória: Linguagem e Sociedade da Universidade Estadual do Sudoeste da Bahia/ PPGM/UESB, Docente do Curso de Enfermagem da Faculdade Independente do Nordeste/ FAINOR.

E-mail: polly_vl@yahoo.com.br

Renato Novaes Chaves - Enfermeiro, Mestrando do Programa de Pós-Graduação em Memória: Linguagem e Sociedade da Universidade Estadual do Sudoeste da Bahia PPGM/UESB, Docente do Curso de Enfermagem da Faculdade de Tecnologia e Ciências / FTC.

E-mail: rnc_novaes@hotmail.com

Elaine dos Santos Santana - Enfermeira, Mestranda do Programa de Pós-Graduação em Memória: Linguagem e Sociedade da Universidade Estadual do Sudoeste da Bahia PPGM/UESB.

E-mail: elaine_137@hotmail.com

Luciana Araújo dos Reis - Fisioterapeuta, Doutora em Ciências da Saúde, UFRN, Docente do Programa de Pós-Graduação em Memória: Linguagem e Sociedade da Universidade Estadual do Sudoeste da Bahia /PPGM/UESB. Vitória da Conquista, BA.

E-mail: lucianauesb@yahoo.com.br 